\title{
On-shell Improved Lattice Gauge Theories
}

\author{
M. Lüscher ${ }^{1}$ and P. Weisz ${ }^{2 \star}$ \\ 1 Deutsches Elektronen-Synchrotron DESY, D-2000 Hamburg, \\ Federal Republic of Germany \\ 2 II. Institut für Theoretische Physik der Universität Hamburg, D-2000 Hamburg 50, \\ Federal Republic of Germany
}

\begin{abstract}
By means of a spectrum conserving transformation, we show that one of the 3 coefficients in Symanzik's improved action can be chosen freely, if only spectral quantities (masses of stable particles, heavy quark potential etc.) are to be improved. In perturbation theory, the other 2 coefficients are however completely determined and their values are obtained to lowest order.
\end{abstract}

\section{Introduction}

Symanzik's improvement programme [1-6] for lattice gauge theories (and other lattice theories) is designed to systematically reduce the cutoff dependence of onand off-shell amplitudes near the continuum limit. Mainly, this is achieved by choosing an improved lattice action, which, at the first level of improvement, is equal to the standard one-plaquette action plus a linear combination of 3 operators ${ }^{1}$ of dimension 6 with perturbatively calculable coefficients $c_{i}\left(g_{0}^{2}\right)\left(g_{0}\right.$ : bare coupling constant, $i=1,2,3$ ). In addition, gauge invariant lattice operators are in general also intrinsically cutoff dependent and, in order to obtain improved correlation functions, must therefore be corrected by subtracting a combination of higher dimensional operators. The necessity of such subtractions has been explicitly demonstrated by Symanzik in the case of the non-linear $\sigma$-model [3. Sect. 4].

For the computation of the coefficients $c_{i}\left(g_{0}^{2}\right)$, the intrinsic cutoff dependence of operators is a potential source of difficulty, because it must be carefully disentangled from the "dynamical" cutoff effects, which are to be cancelled by improving the action. This problem can however be entirely avoided, if only the improvement of spectral quantities (e.g. the static quark-antiquark potential at physical distances) is required. Such quantities are independent of the choice of "interpolating" operator field and it therefore makes no difference whether the operators one uses have or have not been corrected.

In this paper, we show that the requirement of on-shell improvement places only two constraints on the coefficients $c_{i}\left(g_{0}^{2}\right)$ so that without loss one may choose $c_{3}\left(g_{0}^{2}\right)=0$, for example. $c_{1}\left(g_{0}^{2}\right)$ and $c_{2}\left(g_{0}^{2}\right)$ are then completely fixed and can be

\footnotetext{
* Heisenberg foundation fellow

1 By abuse of notation, we use the word "operator" for any Euclidean ( $\mathbb{C}$-number) field, which can be composed from the fundamental gauge field
} 
computed straightforwardly by evaluating two distinct spectral quantities in perturbation theory. We are presently performing such a calculation to one-loop order and shall here only quote the tree level result (Sect. 4).

In view of the rather non-trivial conceptual structure of the improvement programme, we include two preparatory sections, which are slightly more extensive than absolutely necessary. First, we explain what is meant by the (classical) dimension of lattice operators and present an efficient method to compute it (Sect. 2). Improved lattice gauge theories are introduced subsequently, emphasizing the necessity to formulate an improvement condition. In Sect. 4, we establish the main result announced above by constructing a transformation, which shifts the parameters in the improved action, but leaves the spectrum unchanged up to $O\left(a^{4}\right)$ (a: lattice spacing). Apparent discrepancies with previous calculations of the coefficients $c_{i}\left(g_{0}^{2}\right)$ are resolved in Sect. 5 and conclusions are drawn in Sect. 6.

\section{Classical Dimension of Local Lattice Operators}

Consider $\mathrm{SU}(N)$ lattice gauge fields $U(n, \mu)$ living on a four-dimensional hypercubic lattice with sites $n \in \mathbb{Z}^{4}$. A simple example for the kind of operators to be studied below is the plaquette operator

$$
\mathcal{O}(n)=\sum_{\mu, v} \operatorname{Tr}\left\{\mathbb{1}-U(n, \mu) U(n+\hat{\mu}, v) U(n+\hat{v}, \mu)^{-1} U(n, v)^{-1}\right\}
$$

( $\hat{\mu}$ : unit vector in the positive $\mu$-direction). Some characteristic features of $\mathcal{O}(n)$ are

a) $\mathcal{O}(n)$ is a gauge invariant polynomial of link variables $U(m, v)$ located near $n$.

b) $\mathcal{O}(n)$ moves covariantly under space-time translations of the lattice gauge field.

All operators that we shall consider later have these general properties, and no further properties are required to unambiguously define the classical dimension of an operator.

Any given smooth continuum gauge field $A_{\mu}(x), x \in \mathbb{R}^{4}$, can be arbitrarily well approximated by lattice gauge fields $U(n, \mu)$ in the following way. Let " $a$ " denote a small distance and superimpose a lattice on $\mathbb{R}^{4}$ with sites $x=a n, n \in \mathbb{Z}^{4}$. The (continuum) parallel transporter from $a n+a \hat{\mu}$ to $a n$ in the field $A_{\mu}(x)$ is then given by the familiar path ordered exponential ${ }^{2}$

$$
U(n, \mu)=T \exp a \int_{0}^{1} d t A_{\mu}(a n+a \hat{\mu}-t a \hat{\mu}) .
$$

For sufficiently small spacing $a, U(n, \mu)$ will be a slowly varying lattice gauge field "triangulating" $A_{\mu}$, and one expects that lattice operators $\mathcal{O}(n)$ evaluated in this field approximate local (continuum) operators formed from $A_{\mu}$. Indeed, inspecting Eq. (2), one easily establishes the existence of an asymptotic expansion

$$
\mathcal{O}(0) \underset{a \rightarrow 0}{\sim} \sum_{k=0}^{\infty} a^{k} O_{k}(0)
$$

2 We use a notation, where $A_{\mu}$ is an anti-hermitian, traceless $N \times N$-matrix 
where $O_{k}(x)$ is a polynomial of $A_{\mu}(x)$ and its derivatives. Due to the gauge covariance of Eq. (2) and the gauge invariance of $\mathcal{O}(0)$, the $O_{k}$ 's are actually gauge invariant, too.

We now define the dimension of a lattice operator $\mathcal{O}(n)$ to be the smallest $k$ for which $O_{k} \neq 0$. For example, in case of the plaquette operator we have

$$
\begin{gathered}
\mathcal{O}(0)=-\frac{a^{4}}{2} \sum_{\mu, v} \operatorname{Tr}\left\{F_{\mu \nu}(0) F_{\mu \nu}(0)\right\}+O\left(a^{5}\right), \\
F_{\mu \nu}=\partial_{\mu} A_{v}-\partial_{v} A_{\mu}+\left[A_{\mu}, A_{v}\right],
\end{gathered}
$$

so that its dimension is equal to 4 . We emphasize that the dimension of lattice operators does not depend on how precisely one approximates continuum gauge fields by lattice fields. In particular, Eq. (2) could be replaced by

$$
U(n, \mu)=\exp a A_{\mu}(a n)
$$

without affecting the leading term in the expansion (3). For the actual computation of the dimension of complicated lattice operators, Eq. (2) is preferable, however, because it allows us to take full advantage of gauge invariance.

When $\mathcal{O}(n)$ transforms as a scalar field under rotations and reflections of the lattice, the operators $O_{k}$ that can occur in the small " $a$ " expansion (3) are severely restricted. In particular, $O_{k}=0$ for odd $k$, and the lowest operators assume the general form

$$
\begin{gathered}
O_{4}=r_{0} \sum_{\mu, v} \operatorname{Tr}\left(F_{\mu \nu} F_{\mu v}\right), \\
O_{6}=r_{1} \sum_{\mu, v} \operatorname{Tr}\left(D_{\mu} F_{\mu \nu} D_{\mu} F_{\mu v}\right)+r_{2} \sum_{\mu, v, \varrho} \operatorname{Tr}\left(D_{\mu} F_{v \varrho} D_{\mu} F_{v \varrho}\right) \\
+r_{3} \sum_{\mu, v, \varrho} \operatorname{Tr}\left(D_{\mu} F_{\mu \varrho} D_{v} F_{v \varrho}\right)+\sum_{\mu} \partial_{\mu} G_{\mu} .
\end{gathered}
$$

Here, the numbers $r_{i}$ depend on the operator $\mathcal{O}(n)$ considered and

$$
D_{\mu} F_{v \underline{e}}=\partial_{\mu} F_{v e}+\left[A_{\mu}, F_{v e}\right] \text {. }
$$

$G_{\mu}$ is a linear combination of the fields

$$
\sum_{v, \varrho} \operatorname{Tr}\left(F_{v \varrho} D_{\mu} F_{v \varrho}\right), \sum_{v, \varrho} \operatorname{Tr}\left(F_{\mu v} D_{\varrho} F_{\varrho v}\right), \sum_{v} \operatorname{Tr}\left(F_{\mu v} D_{\mu} F_{\mu v}\right),
$$

which we shall never need to work out explicitly. The coefficients $r_{i}$, on the other hand, will be required frequently, and the remainder of this section is therefore devoted to the question of how to compute them efficiently.

Let us begin with the small ' $a$ ' expansion of the simple operator

$$
\mathcal{O}_{\mu \nu}(n)=\operatorname{Tr}\left\{U(n, \mu) U(n+\hat{\mu}, v) U(n+\hat{v}, \mu)^{-1} U(n, v)^{-1}\right\} .
$$

For any given fixed pair of indices $\mu \neq v$, choose the gauge

$$
\begin{array}{ll}
A_{\mu}(x)=0 & \text { for all } x, \\
A_{v}(x)=0 & \text { for all } x \text { with } x_{\mu}=0 .
\end{array}
$$

$\mathcal{O}_{\mu v}(0)$ then reduces to

$$
\mathcal{O}_{\mu v}(0)=\operatorname{Tr}\left\{T \exp a \int_{0}^{1} d t A_{v}(a \hat{\mu}+(1-t) a \hat{v})\right\} .
$$


Furthermore, at $x=0$, we have

so that

$$
\partial_{v}^{p} \partial_{\mu}^{q+1} A_{v}=D_{v}^{p} D_{\mu}^{q} F_{\mu \nu},
$$

$$
\begin{aligned}
A_{v}(a \hat{\mu}+(1-t) a \hat{v})= & a F_{\mu \nu}+\frac{1}{2} a^{2}\left[D_{\mu}+2(1-t) D_{v}\right] F_{\mu \nu} \\
& +\frac{1}{6} a^{3}\left[D_{\mu}^{2}+3(1-t) D_{v} D_{\mu}+3(1-t)^{2} D_{v}^{2}\right] F_{\mu \nu}+O\left(a^{4}\right) .
\end{aligned}
$$

Expanding the exponential in Eq. (11), noting $\operatorname{Tr} A_{v}=0$ and using (13), quickly yields the result

$$
\begin{aligned}
\mathcal{O}_{\mu \nu}(0)= & N+\frac{1}{2} a^{4} \operatorname{Tr}\left(F_{\mu \nu} F_{\mu \nu}\right)+\frac{1}{2} a^{5} \operatorname{Tr}\left[F_{\mu \nu}\left(D_{\mu}+D_{v}\right) F_{\mu \nu}\right] \\
& +\frac{1}{6} a^{6} \operatorname{Tr}\left\{F_{\mu \nu} F_{\mu \nu} F_{\mu \nu}+F_{\mu \nu}\left(D_{\mu}^{2}+\frac{3}{2} D_{\mu} D_{v}+D_{v}^{2}\right) F_{\mu \nu}\right. \\
& \left.+\frac{3}{4}\left(D_{\mu}+D_{v}\right) F_{\mu \nu}\left(D_{\mu}+D_{v}\right) F_{\mu \nu}\right\}+O\left(a^{7}\right) .
\end{aligned}
$$

This is a relation between gauge invariant quantities and it therefore holds for all gauge fields $A_{\mu}$, including those which do not satisfy the gauge condition (10).

More complicated operators than $\mathcal{O}_{\mu \nu}$ can be treated similarly by writing them as a sum of $\mathcal{O}_{\mu \nu}$ 's plus traces of products of gauge covariant plaquette matrices such as

$$
P_{\mu v}(n)=U(n, \mu) U(n+\hat{\mu}, v) U(n+\hat{v}, \mu)^{-1} U(n, v)^{-1}-\mathbb{1} .
$$

Proceeding as above, these can be expanded and one obtains, for example,

$$
\begin{aligned}
P_{\mu \nu}(0)= & a^{2} F_{\mu \nu}+\frac{1}{2} a^{3}\left(D_{\mu}+D_{\nu}\right) F_{\mu \nu} \\
& +\frac{1}{6} a^{4}\left\{3 F_{\mu \nu} F_{\mu \nu}+\left(D_{\mu}^{2}+\frac{3}{2} D_{\mu} D_{\nu}+D_{v}^{2}\right) F_{\mu \nu}\right\}+O\left(a^{5}\right) .
\end{aligned}
$$

Because $P_{\mu \nu}$ 's are always multiplied by other $P_{\varrho \sigma}$ 's, it is not necessary to go beyond $O\left(a^{4}\right)$ in Eq. (16). In this way, all the operators encountered later can be expanded up to order $a^{6}$ with little effort (see Sect. 3.3 for explicit results).

\section{Improved Lattice Gauge Theories}

\section{1. $O\left(a^{2}\right)$ Scaling Violations}

Suppose we are given an $\mathrm{SU}(N)$ lattice gauge theory with action $S[U]$ and bare coupling constant $g_{0}$. Euclidean expectation values of gauge invariant combinations $Q$ of the field $U(n, \mu)$ are defined by

$$
\langle Q\rangle=\frac{1}{Z} \int \mathscr{D}[U] Q[U] e^{-S[U]},
$$


where $Z$ is a normalizing factor such that $\langle 1\rangle=1$ and

$$
\mathscr{D}[U]=\prod_{n, \mu} d U(n, \mu)
$$

[ $d U$ denotes the normalized Haar measure on $\mathrm{SU}(N)]$.

Physically interesting quantities $M$ such as the masses of stable particles and their scattering amplitudes ${ }^{3}$ can (in principle) be extracted from the Euclidean correlation functions of suitable local lattice operators. Initially, when expressed in lattice units, these quantities only depend on $g_{0}$ and a set of external space-like lattice momenta collectively denoted by $k\left(-\pi \leqq k_{i} \leqq \pi \text { for each component } k_{i}\right)^{4}$. In order to study the continuum limit, it is convenient to introduce a lattice spacing " $a$ " by defining scaled quantities $m$ through

$$
m\left(p, g_{0}, a\right)=a^{-\delta} M\left(a p, g_{0}\right),
$$

where $\delta$ is the engineering dimension of $M$ and $p=k / a$ a "physical" momentum.

In a lattice gauge theory with perfect scaling it would be possible to find a function $\bar{g}_{0}(a)$ such that

$$
a \frac{d}{d a} m\left(p, \bar{g}_{0}(a), a\right)=0
$$

for all quantities $m$. For a generic action this will however not be possible and, according to perturbation theory, the best one can achieve is approximate scaling near the continuum limit, i.e. for $a \rightarrow 0$ we then have ${ }^{5}$

$$
a \frac{d}{d a} m\left(p, \bar{g}_{0}(a), a\right)=O\left(a^{2}\right) .
$$

Note that there are many functuions $\bar{g}_{0}(a)$, which lead to approximate scaling. For what follows, it is helpful to remove this ambiguity by choosing once and for all a physical condition, which fixes $\bar{g}_{0}(a)$ uniquely. For example, one may require that the mass gap is equal to $1 \mathrm{GeV}$ for all $a$. Or, more suitable for perturbation theory, that the force between heavy quarks is equal to $\mathrm{Cg}^{2} / 4 \pi \mathrm{GeV}^{2}$ at a distance of $1 \mathrm{GeV}^{-1}[\mathrm{~g}$ a fixed small number, the "renormalized" coupling, and $\left.C=\left(N^{2}-1\right) / 2 N\right]$. Ultimately, it does not matter what condition is chosen, but we shall assume that it is the same for all theories considered and that it has the general form

$$
m_{0}\left(\bar{g}_{0}(a), a\right)=\hat{m}_{0},
$$

where $m_{0}$ is some spectral physical quantity and $\hat{m}_{0}$ a fixed value.

It is possible that different lattice actions $S$ and $S^{\prime}$ yield the same physical amplitudes within an error of order $a^{2 k}$. In more precise terms, this means the following. Any given physical quantity can be computed in the two theories so that one has two sets of functions $m\left(p, g_{0}, a\right)$ and $m^{\prime}\left(p, g_{0}, a\right)$ (the bare coupling constant $g_{0}$ is just a parameter in the action and we are free to denote it by the same symbol

3 I.e. full propagator amputated, connected $n$-point functions evaluated on the (lattice) mass shell

4 Other external length scales may occur but are omitted here for simplicity

5 We use a sloppy notation, where $O\left(a^{2}\right)$ may also stand for a term of order $a^{2}(\ln a)^{r}$ 
in all theories). In particular, $\bar{g}_{0}(a)$ and $\bar{g}_{0}^{\prime}(a)$ may be determined and the approximate physical equivalence of $S$ and $S^{\prime}$ is then expressed through

$$
m\left(p, \bar{g}_{0}(a), a\right)=m^{\prime}\left(p, \bar{g}_{0}^{\prime}(a), a\right)+O\left(a^{2 k}\right) .
$$

Due to universality, a large class of actions are expected to have identical continuum limits and are hence equivalent up to $O\left(a^{2}\right)$. Examples of actions $S^{\prime}$ that are physically indistinguishable from a given action $S$ [i.e. no error term in Eq. (23)] are also easily constructed by local, gauge covariant substitutions of the field variables $U(n, \mu)$. Such transformations affect correlation functions, but do not change spectral quantities. This fact will be exploited in Sect. 4.

\subsection{Improvement Conditions}

The aim of Symanzik's improvement programme is to reduce the $O\left(a^{2}\right)$ scaling violations in Eq. (21) down to $O\left(a^{4}\right)$ by choosing an improved lattice action. Actually, in his study of the $\phi^{4}$-theory [2], Symanzik was able to show that not only spectral quantities can be improved, but also the (properly normalized) $n$-point functions of the fundamental field in momentum space. In the 2-dimensional non-linear $\sigma$-model, the situation turned out to be not so simple, in particular, the $n$-point functions of the lattice spin field $\phi$ could not be improved by merely choosing an improved action [3]. However, the $n$-point functions of a corrected field operator

$$
\boldsymbol{\phi}^{\prime}=\boldsymbol{\phi}+\bar{c}_{1}\left(g_{0}\right)(\boldsymbol{\phi} \cdot \square \boldsymbol{\phi}) \boldsymbol{\phi}+\bar{c}_{2}\left(g_{0}\right) \square \boldsymbol{\phi},
$$

can be improved, provided the coefficients $\bar{c}_{i}$ are chosen appropriately and provided one subtracts certain contact terms from the correlation functions. For gauge theories, no in depth analysis of $O\left(a^{2}\right)$ scaling violations has yet been made and it is not known whether e.g. the improvement of uncorrected (but normalized) Wilson loops is possible to all orders of perturbation theory.

An improvement condition is a statement specifying which quantities are to be improved. In general, different improvement conditions require different improved actions. Moreover, some do not determine the improved action uniquely and still others may be inconsistent as discussed above. Here we shall adopt the "minimal" improvement condition, which requires that the error term in Eq. (21) be reduced to $O\left(a^{4}\right)$ for all low lying energy values $m^{6}$. This includes, in particular, the force between heavy quarks at physical distances and the spectrum of the field excitations in a periodic (space-) box of physical size [8]. Because of the relation between finite size effects on the mass spectrum and scattering amplitudes [9], we expect that the improvement of the energy spectrum implies the improvement of the latter, too. In any case, lattice theories satisfying our condition will be called "on-shell improved."

The scalar field theories for which Symanzik has worked out the improved action are also on-shell improved. His improvement conditions can however be

6 Energy values can be read off from the exponential decay of correlation functions at large times. Equivalently, they can be identified with the eigenvalues $\lambda$ of the transfer matrix $\mathbb{T}$ of [7] through $m=-\frac{1}{a} \ln (\lambda / \Lambda)(\Lambda:$ ground state eigenvalue of $\mathbb{T})$ 
Table 1. List of the sets $\mathscr{S}_{i}$ of elementary loops $\mathscr{C}$ on the lattice. Only the loops of Fig. 1 occur in the improved action. The other loops are generated by the field transformation discussed in Sect. 4

\begin{tabular}{llc}
\hline Set & Description of elements & $\begin{array}{l}\text { No. of } \\
\text { elements/site }\end{array}$ \\
\hline $\mathscr{S}_{0}$ & Plaquettes (Fig. 1a) & 6 \\
$\mathscr{S}_{1}$ & Rectangles (Fig. 1b) & 12 \\
$\mathscr{S}_{2}$ & Parallelograms (Fig. 1c) & 16 \\
$\mathscr{S}_{3}$ & Bent rectangles (Fig. 1d) & 48 \\
$\mathscr{S}_{4}$ & Double plaquettes (Fig. 2a) & 6 \\
$\mathscr{S}_{5}$ & Twisted rectangles (Fig. 2b) & 12 \\
$\mathscr{S}_{6}$ & Bent twisted rectangles (Fig. 2c) & 48 \\
\hline
\end{tabular}

more restrictive. For example, in the $\phi^{4}$-theory, the requirement that all $n$-point functions of the field be improved completely determines the coefficients on the improved action, while some of them remain free, if only on-shell improvement is required (cf. Sect. 4).

\subsection{Ansatz for the Improved Action}

Following [4] the improved action is written as ${ }^{7}$

$$
S[U]=\frac{2}{g_{0}^{2}} \sum_{i=0}^{3} c_{i}\left(g_{0}^{2}\right) \sum_{\mathscr{C} \in \mathscr{S}_{i}} \mathscr{L}(\mathscr{C}),
$$

where the $\mathscr{S}_{i}$ 's denote sets of elementary loops $\mathscr{C}$ on the lattice as described by Table 1 . A given set $\mathscr{S}_{i}$ contains all loops of a definite shape that can be drawn on the lattice. Loops $\mathscr{C}$ that differ by orientation only are considered equal and the weight $\mathscr{L}(\mathscr{C})$ is defined by

$$
\mathscr{L}(\mathscr{C})=\operatorname{Re} \operatorname{Tr}[\mathbb{1}-U(\mathscr{C})],
$$

$U(\mathscr{C})$ being the ordered product of the link variables $U(n, \mu)$ along $\mathscr{C}$. Finally, the coefficients $c_{i}\left(g_{0}^{2}\right)$ are regular at $g_{0}^{2}=0$ and are normalized such that

$$
c_{0}\left(g_{0}^{2}\right)+8 c_{1}\left(g_{0}^{2}\right)+8 c_{2}\left(g_{0}^{2}\right)+16 c_{3}\left(g_{0}^{2}\right)=1 .
$$

This is merely a convention. It could always be enforced by renormalizing $g_{0}$, if it should not hold initially.

The structure of the improved action becomes more transparent if we rewrite it in terms of local lattice operators. Thus, pick a set of operators $\mathcal{O}_{i}(n)$, scalar under rotations and reflections, such that

$$
\sum_{\mathscr{C} \in \mathscr{S}_{i}} \mathscr{L}(\mathscr{C})=\sum_{n} \mathscr{O}_{i}(n)
$$

7 Our notation differs from [4-6] by an interchange of $c_{2}$ with $c_{3}$ 


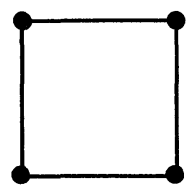

a

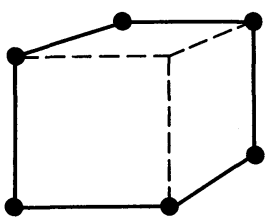

$c$

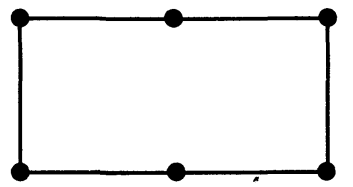

b

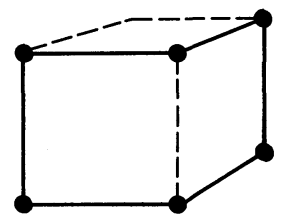

d

Fig. 1a-d. Elementary loops $\mathscr{C}$ on the lattice. a and $\mathbf{b}$ are planar loops, while $\mathbf{c}$ and $\mathbf{d}$ extend in 3 dimensions. Dashed lines are drawn to guide the eye

Table 2. List of the coefficients $r_{i}$ [Eqs. (6) and (7)] that occur in the small " $a$ " expansion (3) of the operators $\mathcal{O}_{i}(n)$ defined in Sect. 3.3

\begin{tabular}{lrrrr}
\hline & $r_{0}$ & $r_{1}$ & $r_{2}$ & $r_{3}$ \\
\hline $\mathcal{O}_{0}$ & $-\frac{1}{4}$ & $\frac{1}{24}$ & 0 & 0 \\
$\mathcal{O}_{1}$ & -2 & $\frac{5}{6}$ & 0 & 0 \\
$\mathcal{O}_{2}$ & -2 & $\frac{1}{3}$ & $\frac{1}{6}$ & $\frac{1}{6}$ \\
$\mathcal{O}_{3}$ & -4 & $\frac{1}{6}$ & 0 & $\frac{1}{2}$ \\
$\mathcal{O}_{4}$ & -1 & $\frac{1}{6}$ & 0 & 0 \\
$\mathcal{O}_{5}$ & 0 & $-\frac{1}{2}$ & 0 & 0 \\
$\mathcal{O}_{6}$ & -4 & $\frac{7}{6}$ & 0 & $-\frac{1}{2}$ \\
\hline
\end{tabular}

Following the method explained in Sect. 2, the classical small " $a$ " expansion (3) of the $\mathcal{O}_{i}$ 's can be worked out. The results are listed in Table $2 .{ }^{8}$ It follows from these that $\mathcal{O}_{0}$ is an operator of dimension 4 and that the operators

$$
\begin{aligned}
& \mathcal{O}_{1}^{\prime}=\mathcal{O}_{1}-8 \mathcal{O}_{0}, \\
& \mathcal{O}_{2}^{\prime}=\mathcal{O}_{2}-8 \mathcal{O}_{0}, \\
& \mathcal{O}_{3}^{\prime}=\mathcal{O}_{3}-16 \mathcal{O}_{0},
\end{aligned}
$$

8 Note that the coefficients $r_{i}$ are independent of the choice of the operators $\mathcal{O}_{i}$ as long as (27) holds. The divergence terms $\sum_{\mu} \partial_{\mu} G_{\mu}$, on the other hand, are dependent and we shall (and may) assume that $\sum_{\mu} \partial_{\mu} G_{\mu}=0$ for all operators $\mathcal{O}_{i}$ 
are all of dimension 6 . In terms of these operators the improved action reads

$$
S=\frac{2}{g_{0}^{2}} \sum_{n}\left\{\mathcal{O}_{0}(n)+\sum_{i=1}^{3} c_{i}\left(g_{0}^{2}\right) \mathcal{O}_{i}^{\prime}(n)\right\} .
$$

In other words, this is the Wilson action plus a linear combination of 3 operators of dimension 6.

Actually, the operators $\mathscr{O}_{i}^{\prime}, i=1,2,3$, from a basis in the space of all operators of dimension 6 , which transform as a scalar field under rotations and reflections. Namely, any such operator $\mathcal{O}$ can be represented by

$$
\mathcal{O}=\sum_{i=1}^{3} \lambda_{i} \mathcal{O}_{i}^{\prime}+\sum_{\mu} \partial_{\mu} \mathscr{G}_{\mu}+\mathscr{R},
$$

where $\mathscr{G}_{\mu}$ and $\mathscr{R}$ are lattice operators of dimension 5 and 8 respectively and $\partial_{\mu}$ denotes a lattice derivative $\left(\partial_{\mu} f(n)=f(n+\hat{\mu})-f(n)\right)$. Thus, up to operators of dimension 8, the Ansatz (29) is the most general one can write down.

As already pointed out, the improvement programme has not yet been shown to work in the case of gauge theories. However, in view of the experience with scalar theories, we are confident (and shall here assume) that there is at least one choice of the coefficients $c_{i}\left(g_{0}^{2}\right)$ such that the theory described by the action (25) is on-shell improved. There is also a further structural property of improvement, which we expect to hold and which we shall make use of in Sect. 4. Namely, suppose $S$ is an on-shell improved action and suppose we add a term

$$
\Delta S=\varepsilon \frac{2}{g_{0}^{2}} \sum_{n} \mathcal{O}(n),
$$

where $\varepsilon$ is infinitesimal and $\mathcal{O}(n)$ a dimension 6 lattice operator scalar under rotations and reflections. In general, this leads to $O\left(a^{2}\right)$ scaling violations in the energy spectrum, and we expect to be able to cancel these effects by adding another term of the form

$$
\widetilde{\Delta} S=-\varepsilon \frac{2}{g_{0}^{2}} \sum_{n} \sum_{i=1}^{3} \Delta_{i}^{\mathcal{O}}\left(g_{0}^{2} ; S\right) \mathcal{O}_{i}^{\prime}(n),
$$

where the coefficients $\Delta_{i}^{\mathscr{O}}\left(g_{0}^{2} ; S\right)$ are computable in perturbation theory (as we shall see in Sect. 4, they are however not uniquely determined). Decomposing the operator $\mathcal{O}(n)$ as in Eq. (30), we may take

$$
\Delta_{i}^{\mathscr{O}}\left(g_{0}^{2} ; S\right)=\lambda_{i}+\Delta_{i}^{\mathscr{R}}\left(g_{0}^{2} ; S\right) .
$$

Now, $\mathscr{R}$ is an operator of dimension 8 and it therefore does not induce $O\left(a^{2}\right)$ effects at the tree level of perturbation theory. Such effects may however show up at one-loop order, but to cancel these, it is sufficient to choose $\Delta_{i}^{\mathscr{R}}\left(g_{0}^{2} ; S\right)=O\left(g_{0}^{2}\right)$, so that altogether we may assume

$$
\Delta_{i}^{\mathscr{O}}\left(g_{0}^{2} ; S\right)=\lambda_{i}+O\left(g_{0}^{2}\right) .
$$

We emphasize again that although the properties just described are highly plausible they really have the status of an unproven hypothesis at present. 


\subsection{Applicability of Ordinary Perturbation Theory}

The only practical way known today to calculate the coefficients $c_{i}\left(g_{0}^{2}\right)$ is by weak coupling expansion about the (locally) pure gauge configurations. Also, all structural statements [Eq. (33) for example] ultimately rely on perturbation theory. Now it can happen that for an action $S$ of the form (25) the classical vacuum $U(n, \mu)=1$ is a false vacuum, i.e. that there are other field configurations with $S<0$. In such a case the perturbation expansion about the pure gauge configurations is not relevant to the continuum limit and any conclusions based on it may be completely misleading. For example, we have found an action, which is on-shell improved at tree level of ordinary (false) perturbation theory, but turns out to be unimproved when expanded about the absolute minima of the action.

Perturbation theory enters our analysis at many places and the conclusions reached therefore apply only in cases where the improved action $S$ is positive for $g_{0}$ $\rightarrow 0$ in the sense that

$$
S[U]>0,
$$

for all lattice gauge fields $U(n, \mu)$, except for (locally) pure gauge configurations. This condition restricts the admissable tree level coefficients $c_{i}(0)$ to some convex domain $P$ in the plane defined by Eq. (27). $P$ could be determined numerically, but it is easier to establish some inequalities, which in many cases of interest are sufficient to decide whether the improved action is positive or not. For example, if

$$
c_{0}+\frac{8}{3} c_{2}<0 \text { or } c_{0}+8 c_{3}<0,
$$

one can show quickly that the action is not positive by exhibiting a field configuration with $S<0$ (details are given in Appendix A). On the other hand, if

$$
c_{0}+8 \hat{c}_{1}+\frac{80}{3} \hat{c}_{2}+32 \hat{c}_{3}>0
$$

where for all $i, \hat{c}_{i}=\frac{1}{2}\left(c_{i}-\left|c_{i}\right|\right)$, one can prove that the action is positive and that ordinary perturbation theory is hence trustworthy. Note, however, that the domain $P$ is certainly larger than the region characterized by the inequality (36).

\section{Isospectral Transformation of Improved Actions}

In order to determine the coefficients $c_{i}$ in the improved action (25), we have computed the energies of a number of excited states of the lattice gauge field enclosed in an $L \times L \times L$ space box with twisted periodic boundary conditions (the twist is introduced for purely technical reasons to simplify the perturbative calculations). We found that in all cases considered the $O\left(a^{2}\right)$ scaling violations could be removed at tree level provided,

$$
\begin{gathered}
c_{1}(0)-c_{2}(0)-c_{3}(0)=-\frac{1}{12}, \\
c_{2}(0)=0 .
\end{gathered}
$$


To this order, Eq. (37) also insures the improvement of the heavy quark potential at physical distances [4]. Equation (38) is however a new result (cf. Sect. 5).

While our computations prove that the relations (37) and (38) are necessary for on-shell improvement, it is not immediately clear that they are also sufficient, because we have only considered a subset of spectral values. This question is resolved by our main result, which is summarized by the following

Theorem. Suppose $S$ is an on-shell improved action of the form (25). Then there exist coefficients $\Delta_{i}\left(g_{0}^{2} ; S\right)$ such that

$$
S+\varepsilon \frac{2}{g_{0}^{2}} \sum_{n} \sum_{i=1}^{3} \Delta_{i}\left(g_{0}^{2} ; S\right) \mathcal{O}_{i}^{\prime}(n)
$$

is an on-shell improved action to first order in $\varepsilon$, and such that

for all actions $S$.

$$
\begin{aligned}
& \Delta_{1}(0 ; S)=1, \\
& \Delta_{2}(0 ; S)=0, \\
& \Delta_{3}(0 ; S)=1,
\end{aligned}
$$

Postponing the proof of the theorem, we note that at one-loop order (and beyond) the coefficients $\Delta_{i}\left(g_{0}^{2} ; S\right)$ must be expected to depend on $S$ so that a non-linear differential equation must be solved to integrate up the infinitesimal transformation (39). According to Eqs. (40), this differential equation however degenerates to a linear one at $g_{0}^{2}=0$ and it immediately follows that all actions $S$ with coefficients $c_{i}\left(g_{0}^{2}\right)$ satisfying (37) and (38) are tree-level improved. Thus, to lowest order $g_{0}^{2}$, the most general on-shell improved action is given by

$$
\begin{gathered}
c_{0}(0)=\frac{5}{3}-24 x, \\
c_{1}(0)=-\frac{1}{12}+x, \\
c_{2}(0)=0, \\
c_{3}(0)=x,
\end{gathered}
$$

where $x$ is a free parameter restricted only by the requirement that the action be positive (cf. Sect. 3.4). In particular, it is necessary that

$$
x<\frac{5}{72} \text {. }
$$

On the other hand, all values $x$ with

$$
|x|<\frac{1}{16},
$$

yield a positive action and are hence allowed. For example, $x=0$ is possible, but $x=\frac{1}{12}$ [which would imply $\left.c_{1}(0)=0\right]$ violates $(42)$ and is therefore excluded. 


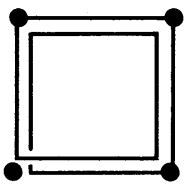

a

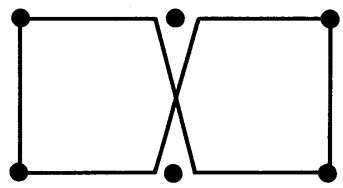

b

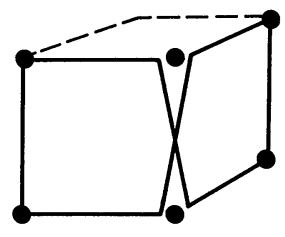

c

Fig. 2a-c. Elementary loops $\mathscr{C}$ generated from the one plaquette loop by the field transformation (46). a winds twice around a single plaquette

Our theorem is also useful at higher orders of $g_{0}^{2}$. For suppose we have already calculated the $c_{i}$ 's up to the $(\ell-1)^{\text {th }}$ loop order and suppose we wanted to compute the next order coefficients $c_{i}^{(\ell)}$. Then, by choosing the parameter $\varepsilon$ in Eq. (39) proportional to $g_{0}^{2 \ell}$, one deduces that these are only determined up to a shift

$$
\begin{aligned}
& c_{1}^{(\ell)} \rightarrow c_{1}^{(\ell)}+y, \\
& c_{2}^{(\ell)} \rightarrow c_{2}^{(\ell)} \\
& c_{3}^{(\ell)} \rightarrow c_{3}^{(\ell)}+y,
\end{aligned}
$$

where $y$ is arbitrary. In particular, we may choose

$$
c_{3}\left(g_{0}^{2}\right)=0,
$$

to all orders in perturbation theory. This choice is the most convenient one for weak coupling calculations, but we emphasize that for Monte Carlo simulations it may be advantageous to take $c_{3} \neq 0$ to keep away from phase transitions at intermediate values of $g_{0}^{2}$. Incidentally, we note that Wilson [10] also chose $c_{3}=0$ for his improved action for a reason not disclosed to the reader.

We now proceed to prove the theorem. Essentially, the idea is to invent a transformation $\Phi$ of lattice gauge fields $U(n, \mu)$, which does not affect the energy spectrum but which changes the improved action by an additive term of dimension 6. Allowing for an error of order $a^{4}$, the transformed improved action may then be replaced by the standard improved action (25) with new coefficients $c_{i}$ so that altogether we have constructed a transformation, which shifts the $c_{i}$ 's, but which does not generate $O\left(a^{2}\right)$ scaling violations.

$\Phi$ maps any given lattice gauge field $U(n, \mu)$ onto a new field $\tilde{U}(n, \mu)$. In order to preserve the basic structure of the action, this mapping should be local and invariant under rotations and reflections of the lattice. We are thus led to make the Ansatz

$$
\tilde{U}(n, \mu)=e^{\varepsilon X_{\mu}(n)} U(n, \mu),
$$

where $\varepsilon$ is infinitesimal and $X_{\mu}(n)$ the anti-hermitian traceless part of another field $Y_{\mu}(n)$ :

$$
\begin{aligned}
& X_{\mu}(n)=Y_{\mu}(n)-Y_{\mu}(n)^{+}-1 / N \operatorname{Tr}\left[Y_{\mu}(n)-Y_{\mu}(n)^{+}\right], \\
Y_{\mu}(n)= & \frac{1}{4} \sum_{v}\left\{U(n, v) U(n+\hat{v}, \mu) U(n+\hat{\mu}, v)^{-1} U(n, \mu)^{-1}\right. \\
& \left.-U(n, \mu) U(n-\hat{v}+\hat{\mu}, v)^{-1} U(n-\hat{v}, \mu)^{-1} U(n-\hat{v}, v)\right\} .
\end{aligned}
$$




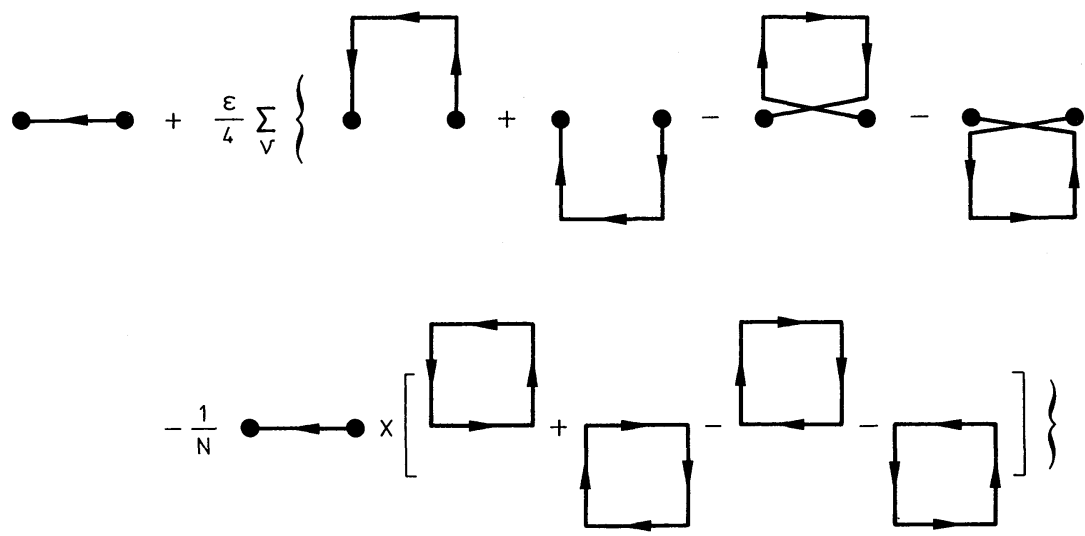

Fig. 3. Graphical representation of $\tilde{U}(n, \mu)$ [cf. Eq. (46)]. The vertices $n$ and $n+\hat{\mu}$ are indicated by dots, link variables by arrows and the whole drawing is in the $(\mu, v)$-plane

The transformed field $\tilde{U}(n, \mu)$ is shown graphically in Fig. 3. Note that it depends on the old field $U(n, \mu)$ in a gauge covariant fashion.

In the classical continuum limit one finds (cf. Sect. 2)

and $\Phi$ reduces to

$$
X_{\mu}=\frac{1}{2} a^{3} \sum_{v} D_{v} F_{v \mu}+O\left(a^{4}\right)
$$

$$
A_{\mu} \rightarrow A_{\mu}+\frac{\varepsilon}{2} a^{2} \sum_{v} D_{v} F_{v \mu}+O\left(a^{3}\right)
$$

It follows that for any lattice operator $\mathcal{O}$ with dimension $d$ we have $\mathcal{O}[\tilde{U}]=\mathcal{O}[U]+\varepsilon \mathscr{P}[U]$, where $\mathscr{P}$ is an operator of dimension $d+2$. In particular, the improved action (29) transforms as

$$
S[\tilde{U}]=S[U]+\varepsilon \frac{2}{g_{0}^{2}} \sum_{n}\left\{\mathscr{P}_{0}(n)+\sum_{i=1}^{3} c_{i}\left(g_{0}^{2}\right) \mathscr{P}_{i}(n)\right\},
$$

$\mathscr{P}_{0}$ and the $\mathscr{P}_{i}$ 's being operators of dimension 6 and 8 , respectively.

We are now ready to complete the proof of the theorem. Thus, suppose $S$ is an improved action of the form (25). As discussed at the end of Sect. 3.3, we may replace $S$ by

$$
S-\varepsilon \frac{2}{g_{0}^{2}} \sum_{n}\left\{\mathscr{P}_{0}(n)+\sum_{i=1}^{3} c_{i}\left(g_{0}^{2}\right) \mathscr{P}_{i}(n)-\sum_{i=1}^{3} \tilde{\Delta}_{i}\left(g_{0}^{2} ; S\right) \mathcal{O}_{i}^{\prime}(n)\right\}
$$

without affecting the energy spectrum except perhaps at order $a^{4}$. In Eq. (50), the coefficients $\tilde{\Delta}_{i}$ are given by

$$
\tilde{\Delta}_{i}\left(g_{0}^{2} ; S\right)=\Delta_{i}^{\mathscr{P} 0}\left(g_{0}^{2} ; S\right)+\sum_{k=1}^{3} c_{k}\left(g_{0}^{2}\right) \Delta_{i}^{\mathscr{P}_{k}}\left(g_{0}^{2} ; S\right) .
$$

Secondly, we perform a substitution of integration variables in the functional integral (17) replacing $U(n, \mu)$ by $\tilde{U}(n, \mu)$. Because this change of variables is local, 
the exponential decay of correlation functions of local operators is not affected and the energy spectrum is exactly the same as before. The action, however, changes according to Eq. (49), and in addition there is a Jacobian, which is calculated in Appendix B. Collecting all terms, the new action reads

$$
S+\varepsilon \frac{2}{g_{0}^{2}} \sum_{n}\left\{-2 C g_{0}^{2} \mathcal{O}_{0}(n)+\sum_{i=1}^{3} \tilde{\Delta}_{i}\left(g_{0}^{2} ; S\right) \mathcal{O}_{i}^{\prime}(n)\right\},
$$

where $C=\left(N^{2}-1\right) / 2 N$. Finally, we replace $g_{0}^{2}$ by $g_{0}^{2}-\varepsilon 2 C g_{0}^{4}$, after which the action (52) assumes the form (39) with

$$
\Delta_{i}\left(g_{0}^{2} ; S\right)=\tilde{\Delta}_{i}\left(g_{0}^{2} ; S\right)+2 C g_{0}^{2}\left(1-g_{0}^{2} \frac{\partial}{\partial g_{0}^{2}}\right) c_{i}\left(g_{0}^{2}\right)
$$

Since all transformations applied conserve the energy spectrum up to at most an error of order $a^{4}$, we have thus generated a new on-shell improved action. It remains to show that the coefficients $\Delta_{i}\left(g_{0}^{2} ; S\right)$ satisfy Eqs. (40). Using Eq. (33), we have

$$
\Delta_{i}(0 ; S)=\Delta_{i}^{\mathscr{P} 0}(0 ; S)=\lambda_{i},
$$

where the $\lambda_{i}$ 's are to be determined from the classical expansion (30) of the operator $\mathscr{P}_{0}$. To this end, we first recall that $\mathscr{P}_{0}$ is obtained from the single plaquette operator $\mathcal{O}_{0}$ by replacing $U(n, \mu)$ by $\widetilde{U}(n, \mu)$ and subtracting the old $\mathcal{O}_{0}$. Thus, with the help of Fig. 3, one finds

$$
\mathscr{P}_{0}=\frac{1}{2}\left\{\mathcal{O}_{1}+\mathcal{O}_{3}-2 \mathcal{O}_{4}-\mathcal{O}_{5}-\mathcal{O}_{6}\right\},
$$

where here and below divergence terms and operators of dimension 8 are neglected. Next, from Table 2 it follows that

$$
\begin{aligned}
& \mathcal{O}_{4}=4 \mathcal{O}_{0}, \\
& \mathcal{O}_{5}=8 \mathcal{O}_{0}-\mathcal{O}_{1}, \\
& \mathcal{O}_{6}=32 \mathcal{O}_{0}-\mathcal{O}_{3},
\end{aligned}
$$

and hence $\mathscr{P}_{0}=-24 \mathcal{O}_{0}+\mathcal{O}_{1}+\mathcal{O}_{3}$. In other words, $\lambda_{1}=\lambda_{3}=1$ and $\lambda_{2}=0$ as required.

\section{Comparison with Earlier Calculations of Improved Actions}

A computation of the coefficients $c_{i}\left(g_{0}^{2}\right)$ to lowest order was first attempted in [4] and the following two relations were obtained:

$$
\begin{gathered}
c_{1}(0)=-\frac{1}{12}, \\
c_{2}(0)+c_{3}(0)=0 .
\end{gathered}
$$

Later, Curci et al. [6] provided an argument for

$$
c_{2}(0)=c_{3}(0)=0
$$


by studying the heavy quark potential at one-loop order. Equation (59) was also derived from a purely classical consideration in [5]. Taken together, the conclusion was that the choice

$$
c_{0}(0)=\frac{5}{3}, \quad c_{1}(0)=-\frac{1}{12}, \quad c_{2}(0)=c_{3}(0)=0
$$

is necessary for improvement.

At first sight, there seems to be a discrepancy with our result, which asserts that there is a whole one-parameter family of tree level improved actions of which (60) is but one member [cf. Eqs. (41)]. The origin of this mismatch is that the authors of [4-6] use improvement conditions, which differ from the one adopted here (Sect. 3.2). It is instructive to trace in each case how the improvement condition influences the outcome of the calculations. In [4], for example, the requirement was to improve all physical size Wilson loops. This includes in particular the heavy quark potential at physical distances $L$, which to lowest order of perturbation theory is given by

$$
V(L)=-C \frac{g_{0}^{2}}{4 \pi L}\left\{1+3 \frac{a^{2}}{L^{2}}\left[c_{1}(0)-c_{2}(0)-c_{3}(0)+\frac{1}{12}\right]+O\left(\frac{a^{4}}{L^{4}}\right)\right\}
$$

$\left(C=\left(N^{2}-1\right) / 2 N\right.$ as before). Now we can see that while the improvement of all Wilson loops leads to Eqs. (57) and (58), the single relation (37) already insures the improvement of the quark potential and this would in fact be the only result that can be extracted from the calculations of [4] once one adopts our on-shell improvement condition.

In their work, Curci et al. consider the Fourier transform $\tilde{V}(\mathbf{p})$ of the heavy quark potential at one-loop order. Besides other $O\left(a^{2}\right)$ contributions, there are two terms proportional to

$$
a^{2} \ln \left(a^{2} \mathbf{p}^{2}\right) \quad \text { and } \quad a^{2}\left(\mathbf{p}^{2}\right)^{-2} \sum_{i=1}^{3} p_{i}^{4} \ln \left(a^{2} \mathbf{p}^{2}\right) .
$$

If one requires $\tilde{V}(\mathbf{p})$ to be improved, the coefficients of both terms must vanish and, in conjunction with Eqs. (57) and (58), this yields $c_{2}(0)=c_{3}(0)=0$. However, from the point of view of our improvement condition, we do not expect $\tilde{V}(\mathbf{p})$ to be improved, because it is a sum of energy values $V(L)$, which also includes energies of the order of the cutoff $(V(L)$ for $L=a$, for example). On the other hand, for physical $L$ the $O\left(a^{2}\right)$ contributions to the quark potential at one-loop order are proportional to $a^{2} L^{-3} \ln L / a$ and $a^{2} L^{-3}$. Now it turns out that the first term disappears as soon as (37) holds, and no further relation for the tree-level coefficients $c_{i}(0)$ is obtained from the second term, because it can be made to vanish by adjusting the one-loop coefficients. Summing up, we have found that also at one-loop order Eq. (37) is in fact the only constraint on the coefficients $c_{i}(0)$, which is implied by the improvement of the quark potential at physical distances. In the framework of our improvement condition, Eq. (38) is therefore a new result. 
In [5] a purely classical argument in favour of Eqs. (60) was presented. The starting point was the small " $a$ " expansion of the improved action $S$, which reads

$$
\begin{aligned}
S= & -\frac{1}{2 g_{0}^{2}} \int d^{4} x\left\{\sum_{\mu, v} \operatorname{Tr}\left(F_{\mu \nu} F_{\mu \nu}\right)-\frac{2}{3} c_{2} a^{2} \sum_{\mu, v, \varrho} \operatorname{Tr}\left(D_{\mu} F_{v \varrho} D_{\mu} F_{v \varrho}\right)\right. \\
& -\left(2 c_{1}-2 c_{3}+\frac{1}{6}\right) a^{2} \sum_{\mu, v} \operatorname{Tr}\left(D_{\mu} F_{\mu \nu} D_{\mu} F_{\mu \nu}\right) \\
& \left.-\left(\frac{2}{3} c_{2}+2 c_{3}\right) a^{2} \sum_{\mu, v, \varrho} \operatorname{Tr}\left(D_{\mu} F_{\mu \varrho} D_{v} F_{v \varrho}\right)+O\left(a^{4}\right)\right\} .
\end{aligned}
$$

The classical action may be considered a generating functional for the vertex functions at tree level and one may therefore expect them to be improved, if the $O\left(a^{2}\right)$ terms in Eq. (62) are absent. This is the case if and only if the coefficients $c_{i}(0)$ assume the values (60). On the other hand, for the on-shell improved actions (41), Eq. (62) reduces to

$$
S=-\frac{1}{2 g_{0}^{2}} \int d^{4} x\left\{\sum_{\mu, v} \operatorname{Tr}\left(F_{\mu \nu} F_{\mu \nu}\right)-2 c_{3}(0) a^{2} \sum_{\mu, v, \varrho} \operatorname{Tr}\left(D_{\mu} F_{\mu \varrho} D_{v} F_{v e}\right)+O\left(a^{4}\right)\right\},
$$

so that at the classical level on-shell improvement apparently means the improvement of the action for solutions of the equations of motions only. ${ }^{9}$

We fin ally remark that Symanzik was quite aware of the possibility to shift parameters in the improved action by making use of the Schwinger-Dyson equations. He has, however, never considered doing so at the tree level, probably because he primarily wanted to improve the correlation functions of the fundamental lattice field without explicit subtractions of the field operator.

\section{Concluding Remarks}

From a practical point of view, our main results are

a) that one coefficient in the improved action remains free if only spectral quantities are to be improved, and

b) that the most general tree level on-shell improved action is characterized by

$$
c_{1}(0)-c_{3}(0)=-\frac{1}{12} \text { and } c_{2}(0)=0 .
$$

(We remind the reader that the improved action must also be positive. See Sect. 3.4.)

One particularly natural choice of the undetermined parameter in the action is

$$
c_{3}\left(g_{0}^{2}\right)=0
$$

not only because perturbative calculations are greatly simplified, but also because in addition to the spectral quantities many other quantities get improved as well, at least to lowest order of perturbation theory.

One may also try to fix the free coefficient in the action by adding a further improvement condition, for example that all physical size (and properly norma-

9 We do not know of an independent argument to this effect and we are therefore unable to prove Eqs. (37) and (38) by inverting the above reasoning 
lized) Wilson loops should be improved. However, one must be very careful not to run into inconsistencies, especially so beyond the tree level. The danger is that at some stage one gets more independent equations than there are free parameters in the action. For this reason we prefer to stick to spectral quantities, which are anyhow the only objects of physical significance.

\section{Appendix A: Positivity of Improved Actions}

We first show that the improved action $S$ cannot be positive if $c_{0}+\frac{8}{3} c_{2}<0$. To this end, choose

$$
U(n, \mu)=\left[\begin{array}{lll}
(-1)^{s_{\mu}} & & 0 \\
& (-1)^{s_{\mu}} & \\
& & 1 \\
& & \ddots
\end{array}\right], \quad s_{\mu}=\sum_{v=0}^{\mu-1} n_{v} .
$$

The sums

$$
\sum_{i}=\sum_{\mathscr{C} \in \mathscr{S}_{i}} \mathscr{L}(\mathscr{C})
$$

are then easily evaluated and one finds

$$
\sum_{0}=24 N_{s}, \quad \sum_{1}=\sum_{3}=0, \quad \sum_{2}=64 N_{s},
$$

where $N_{s}$ is the number of sites in the lattice (we assume periodic boundary conditions for convenience). It follows that

$$
S=\frac{2}{g_{0}^{2}} 24 N_{s}\left(c_{0}+\frac{8}{3} c_{2}\right)<0,
$$

i.e. the action is not positive.

Next, suppose $c_{0}+8 c_{3}<0$. Then, replacing $s_{\mu}$ in (A1) by

$$
s_{\mu}=\delta_{\mu 3} \sum_{\mu=0}^{2} n_{v},
$$

we have for this configuration

$$
S=\frac{2}{g_{0}^{2}} 12 N_{s}\left(c_{0}+8 c_{3}\right)<0,
$$

and it follows again that the action is not positive.

Finally, we prove that the inequality (36) implies the positivity of the improved action. The basic ingredient in the proof is the following corollary of the Cauchy-Schwarz inequality:

Lemma. Suppose $U$ and $V$ are two unitary matrices. Then

$$
\operatorname{Re} \operatorname{Tr}(1-U V) \leqq 2 \operatorname{Re} \operatorname{Tr}(1-U)+2 \operatorname{Re} \operatorname{Tr}(1-V),
$$

where the equality holds if and only if $U=V=1$. 
Now let $\mathscr{C}$ be any rectangular loop as shown in Fig. 1b. Writing it as product of two plaquette loops $\mathscr{C}_{1}$ and $\mathscr{C}_{2}$, the lemma yields $\mathscr{L}(\mathscr{C}) \leqq 2 \mathscr{L}\left(\mathscr{C}_{1}\right)+2 \mathscr{L}\left(\mathscr{C}_{2}\right)$. Summing over all rectangles, we have

Similarly, one derives

$$
\sum_{1} \leqq 8 \sum_{0}
$$

$$
\sum_{2} \leqq \frac{80}{3} \sum_{0}, \quad \sum_{3} \leqq 32 \sum_{0} .
$$

These inequalities together with $\sum_{i} \geqq 0$ imply

$$
S \geqq \frac{2}{g_{0}^{2}}\left(c_{0}+8 \hat{c}_{1}+\frac{80}{3} \hat{c}_{2}+32 \hat{c}_{3}\right) \sum_{0} .
$$

Thus, if (36) holds, the improved action is bounded from below by a positive multiple of the Wilson action and is hence positive.

\section{Appendix B: Computation of the Jacobian of the Transformation $\Phi$}

The calculation of the Jacobian of $\Phi$ can be greatly simplified with the help of a lemma from the general theory of Lie group (e.g. [11, Chap. X]). Thus, let $G$ be a Lie group with Lie algebra $(5, d \mu(g)$ a (right-) invariant measure on $G$, and $\Phi: G \rightarrow G$ a differentiable invertible mapping. For every $g \in G$ a linear mapping $\varphi_{g}: \mathfrak{G} \rightarrow \mathfrak{G}$ can be defined through

$$
\Phi\left(e^{t Z} g\right) \cdot \Phi(g)^{-1}=\exp \left\{t \varphi_{g}(Z)+O\left(t^{2}\right)\right\}
$$

for all $Z \in \mathbf{6}$. The lemma alluded to above then reads

Lemma. For every integrable function $f: G \rightarrow \mathbb{C}$ the following substitution rule holds:

$$
\int_{G} d \mu(g) f(g)=\int_{G} d \mu(g)\left|\operatorname{det} \varphi_{g}\right| f(\Phi(g)) .
$$

In our case, the group $G$ is equal to the set of all lattice gauge fields, i.e. it is isomorphic to $[\mathrm{SU}(N)]^{4} N_{s}$, where $N_{s}$ is the number of sites in the lattice. Correspondingly, the Lie algebra $\left(\mathfrak{G}\right.$ is identified with the set of all fields $Z_{\mu}(n)$ of traceless anti-hermitian $N \times N$-matrices. For example, if the group element $g$ stands for the field $U(n, \mu)$, we have the correspondence

$$
e^{t Z} \cdot g \leftrightarrow e^{t Z_{\mu}(n)} \cdot U(n, \mu) .
$$

Inserting this into the definition (46) of the transformation $\Phi$, it is trivial to compute $\varphi_{g}$, and one finds to first order in $\varepsilon$,

$$
\operatorname{det} \varphi_{g}=1+\varepsilon \frac{2}{N}\left(N^{2}-1\right) \sum_{\mathscr{C} \in \mathscr{S}_{0}}[\mathscr{L}(\mathscr{C})-N]
$$

This is in fact the Jacobian we are looking for, because the integral (B2) has exactly the form of the functional integral (17), if we identify $d \mu(g)$ with the measure $\mathscr{D}[U]$ [Eq. (18)]. 


\section{References}

1. Symanzik, K.: Some topics in quantum field theory. In: Mathematical problems in theoretical physics. Schrader, R. et al. (eds.). Lecture Notes in Physics, Vol. 153, Berlin, Heidelberg, New York: Springer 1982

2. Symanzik, K.: Continuum limit and improved action in lattice theories. I. Principles and $\phi^{4}$ theory. Nucl. Phys. B226, 187 (1983)

3. Symanzik, K.: Continuum limit and improved action in lattice theories. II. $O(N)$ nonlinear sigma model in perturbation theory. Nucl. Phys. B226, 205 (1983)

4. Weisz, P.: Continuum limit improved lattice action for pure Yang-Mills theory. I. Nucl. Phys. B212, 1 (1983)

5. Weisz, P., Wohlert, R.: Continuum limit improved lattice action for pure Yang-Mills theory. II. Nucl. Phys. B236, 397 (1984)

6. Curci, G., Menotti, P., Paffuti, G.: Symanzik's improved lagrangian for lattice gauge theory. Phys. Lett. 130 B, 205 (1983)

7. Lüscher, M., Weisz, P.: Definition and general properties of the transfer matrix in continuum limit improved lattice gauge theories. DESY-preprint 84-018 (1984). Nucl. Phys. B (to be published)

8. Lüscher, M.: Some analytic results concerning the mass spectrum of Yang-Mills gauge theories on a torus. Nucl. Phys. B 219, 233 (1983)

9. Lüscher, M.: On a relation between finite size effects and elastic scattering processes. Cargèse lecture notes, DESY-preprint 83-116 (1983). New York: Plenum Press (to be published)

10. Wilson, K.G.: Monte-Carlo calculations for lattice gauge theory. In: Recent developments in gauge theories, G. 't Hooft et al. (eds.). (Cargèse 1979), New York: Plenum Press 1980

11. Helgason, S.: Differential geometry and symmetric spaces. New York: Academic Press 1962

Communicated by G. Mack

Received April 5, 1984 
